\title{
Facial Expressions Based Emotion Recognition through Feature Fusion Approach
}

\author{
Bharati Dixit $^{1+}$ and Arun Gaikwad ${ }^{2}$ \\ ${ }^{1}$ Sinhgad College of Engineering, Pune, India \\ ${ }^{2}$ Zeal College of Engineering and Research, Pune, India
}

\begin{abstract}
Human computer interaction is need of the time and has wide application range. Automatic emotion recognition is one of the important tasks which can be implemented through HCI systems. Facial expressions based non-verbal approach of emotion recognition is experimented for the work. Extracted features are fusion of features obtained through LDN and PCA. Extracted features are provided to feed forward neural network for emotion classification. The experimentation is carried out for CK+ and JAFFE database. The emotion recognition accuracy obtained for CK+ database is $82.07 \%$ and for JAFFE database is 84.22\%. FAR - false acceptance rate and FRR - false rejection rate obtained for CK+ database is $4.18 \%$ and $17.92 \%$ respectively. FAR and FRR obtained for JAFFE database is $3.60 \%$ and $15.77 \%$ respectively.
\end{abstract}

Keywords: local directional number, facial expression analysis, principle component analysis.

\section{Introduction}

Human computer interaction based applications make human life better. Automatic emotion recognition has many applications like e-learning, behavior analysis, cognitive assessment, pain monitoring, driver alert systems etc. Emotion recognition is carried out using verbal and non-verbal approaches. There are many non-verbal approaches like facial expression based emotion analysis, using EEG-Electro Encephalograph signals, through posture and gesture recognition etc. The results presented in this paper are based on facial expressions based approach.

Feature extraction from captured images is very important step in the process of emotion recognition. Three approaches of feature extraction are commonly used. These approaches are (i) appearance based methods - methods like PCA, LDA-Linear discriminant analysis etc. which are based on global features are part of this category (ii) model based methods - methods like LBP-Local Binary Pattern, LDN-Local Directional Number, graph based methods etc. designed on local features are part of this category. (iii) Hybrid methods - the techniques which make combination/fusion of local and global features are part of this category.

The method experimented for this work is hybrid in nature which is designed using fusion of features obtained through LDN - local directional number and PCA - principal component analysis. FFNN- feed forward neural network is used as classifier. The method is experimented on JAFFE database and CK+ database.

The paper is developed under different sections. Section 2 describes relevant studies. Section 3 discusses implementation methodology. Section 4 provides details of experimentation and results. Section 5 is conclusion followed by references in Section 6.

\section{Relevant Studies}

Papers from renowned repositories are studied and key points of some of the relevant papers are presented in this section.

\footnotetext{
+ Corresponding author.Tel.: + 9102030273400.
}

E-mail address: dixit.bharati@gmail.com. 
Adin Ramirez et al. [1] proposes a novel local feature descriptor for face analysis called as LDN-local directional number pattern. LDN encodes the directional information of the face's textures in compact way. Structure of each micro-pattern is computed as directional information and assigned a number called directional number. Face is divided into different regions. LDN is computed for each region and concatenated as feature vector. The feature vector serves as input to classifier. Descriptor performs consistently under illumination, noise and time lapse variations. The descriptor is tested with different masks to analyze its performance in different face analysis tasks.

Li Zhang et al. [2] proposes a unique facial expression recognition system with a variant of evolutionary fire- fly algorithm for feature optimization. A modified Local Binary Pattern descriptor is proposed to produce an initial discriminative face representation. A variant of the firefly algorithm is proposed to perform feature optimization. The proposed evolutionary firefly algorithm exploits the spiral search behaviour of moths and attractiveness search actions of fireflies to achieve early convergence of the Levyflight firefly algorithm (LFA) and the moth-flame optimization (MFO) algorithm. This system achieves a superior performance, and outperforms other state-of-the-art feature optimization methods and related facial expression recognition models by a significant margin.

Ligang Zhang et al. [3] discusses facial expressions as communication channel for conveying emotions and features of facial element and muscle movements are used to extract static, dynamic and geometric characteristics of facial expressions. The technique outperforms many state of the art techniques and experimented on CK - Cohn Kanade Database. Gabor patches are used to extract facial features and correct recognition rate achieved is more than $90 \%$.

Sailee R Salkar et al. [4] has worked with LDN - Local directional Number based feature extraction method to experiment for scenarios where illumination varies. Experimentation is carried out for YALE, PIE and ORL database. Emotion recognition rate is obtained as more than $70 \%$.

Happy S. L. et al. [5] uses salient facial patches for feature extraction. A novel framework of facial expression recognition based on selected facial patches is proposed and experimented on CK+ and JAFFE database. Due to use of selected patches the time required for expression recognition is less and accuracy achieved as more than $95 \%$ for training using fused data samples.

Anima Majumdar [6] has proposed an emotion recognition model based on twenty six dimensional geometric feature vector. Model uses intermediate Kohonen self-organizing map layer as classifier. A comparative study with Radial basis function, Multi-layer perceptron and Support vector machine is carried out.

\section{Implementation Methodology}

The logic and different stages of implementation of emotion detection system are as shown in Fig. 1. Fusion of features [7] obtained from PCA and LDN is carried out and further provided as input to feed forward neural network classifier. The system is trained using $66 \%$ images of database and rest $33 \%$ images are used as test images.

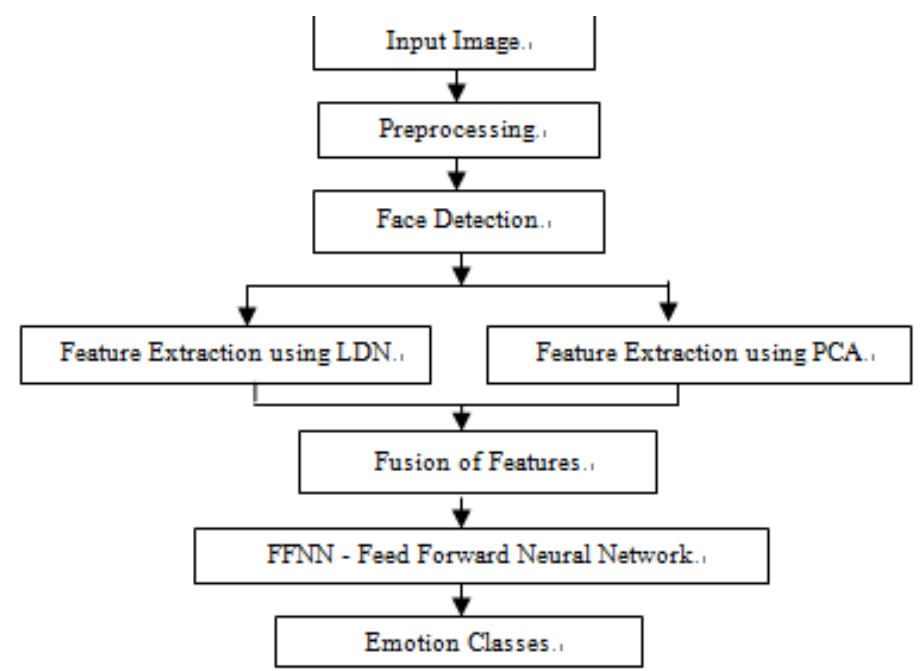

Fig. 1: Implementation stages of emotion detection system. 
Principal component analysis provides low dimensional subspace representation of images and carries the benefit that knowledge about geometry and reflectance of faces is not required. PCA decomposes face image into Eigen faces which work as initial principle components of feature vector.

LDN - Local directional number, feature descriptor is computed by convolving the original image with the mask to extract the edge response images. The most positive and the most negative directions are used to encode the information. The six bit binary code is generated and called as LDN image. The face image is divided into several regions and LDN is computed for each region. Histogram of each region is computed and all these histograms are concatenated to form a feature vector.

Fusion of features obtained through PCA and LDN forms the final feature vector. The final feature vector comprises features like entropy, no. of components like eye, mouth, nose etc., area i.e. total no. of pixels in the region of interest, orientation in terms of lines and edges and contrast for visual perception.

\section{Experimental Results and Performance Analysis}

Experimentation is carried out on JAFFE - Japanese Female Face Emotion Database and CK+ database which is extended Cohn kanade database of emotions with details of action coded units [8]. Experimentation on 184 images of JAFFE database and 283 images of CK+ database is carried out for six emotions like happy, surprise, disgust, anger, sad and fear. Accuracy, false acceptance rate, false rejection rate are used as performance parameters. The simulation tool used for experimentation is MatLab. Some of the screenshots obtained during experimentation are shown in Fig. 2 to Fig. 5.

Original images from JAFFE database [9] and CK+ database [10] are fed to the emotion recognition system. Some of the original images are shown in Fig. 2.

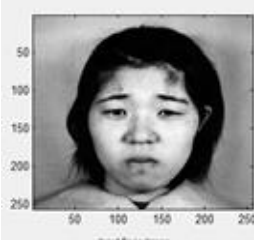

(a)

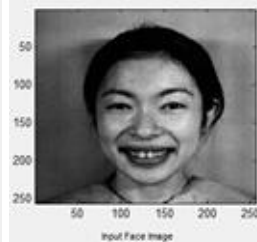

(b)

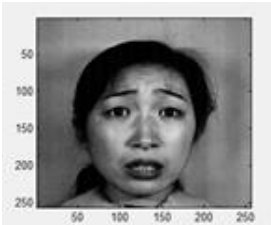

(c)

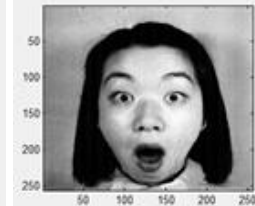

(d)

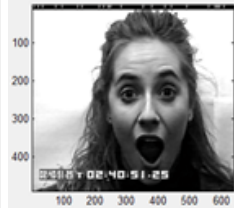

(e)

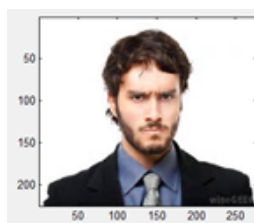

(f)

Fig. 2: Face input images from JAFFE Database (a, b, c and d) and CK+ database (e, f).

Face region is detected from input image. Localization of face region is shown in Fig. 3.

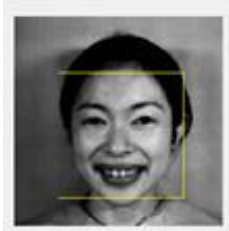

(a)

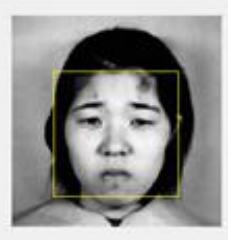

(b)

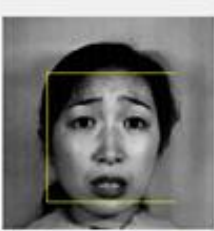

(c)

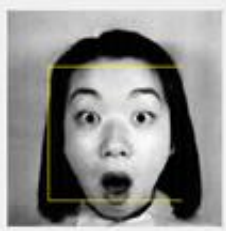

(d)

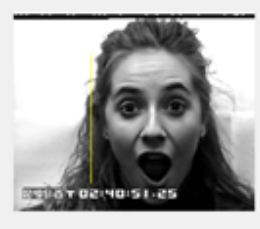

(e)

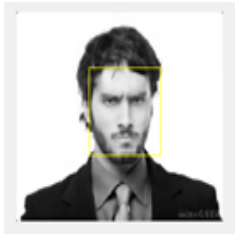

(f)

Fig. 3: Pre-processed face Images - JAFFE Database (a, b, c and d) and CK + database (e, f).

Principal component analysis is applied on face region. The output images are as shown in Fig. 4

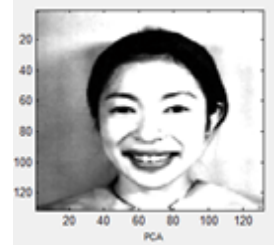

(a)

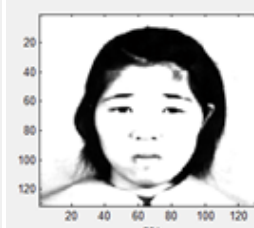

(b)

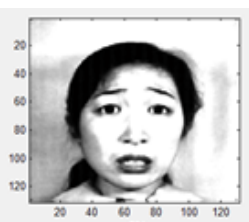

(c)

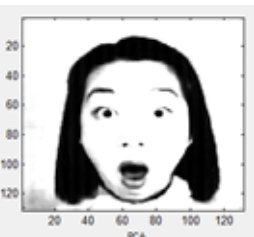

(d)

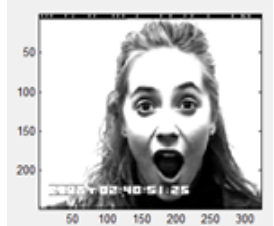

(e)

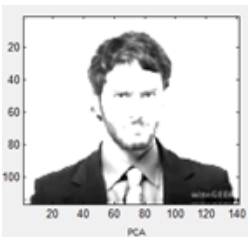

(f)

Fig. 4: Images after applying PCA - JAFFE Database (a, b, c and d) and CK+ database (e, f).

LDN - Local directional number is applied on face region of images. The result is as shown in Fig. 5. 


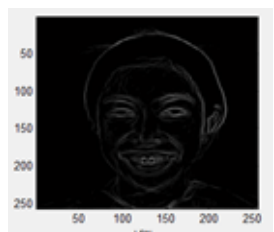

(a)

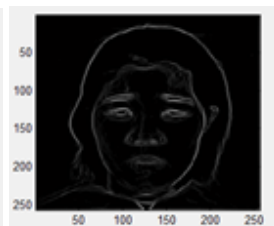

(b)

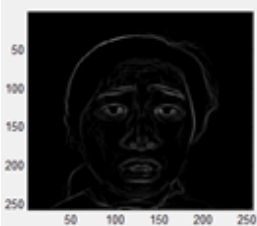

(c)

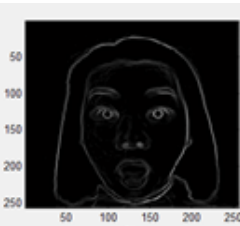

(d)

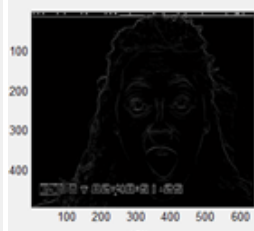

(e)

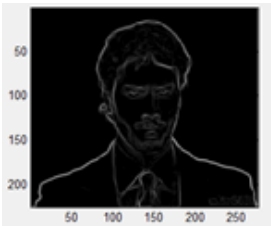

(f)

Fig. 5: Images after applying LDN - JAFFE Database (a, b, c and d) and CK+ database (e, f).

The fusion of features from PCA and LDN is carried out and available in the form of feature vector which is fed to FFNN classifier. Outcome of the system is in the form of emotion classes such as anger, disgust, fear, sad, happy and surprise. The detailed analysis is presented in the form of confusion matrix. Confusion matrix is represented in Table 1 and Table 2 for CK+ database and JAFFE database respectively. Performance parameters such as accuracy, FAR- false acceptance rate and FRR- false rejection rate are calculated from confusion matrix and tabulated in Table 3. Various emotions are coded as AN - anger, DI disgust, FE-Fear, HA-Happy, SA-sad and SU-Surprise while tabulation.

Table 1: Confusion matrix for $\mathrm{CK}+$ Database

\begin{tabular}{|c|c|c|c|c|c|c|c|}
\hline $\begin{array}{c}\text { Emotions(Actual emotions row wise and predicted } \\
\text { emotions as column wise) }\end{array}$ & AN & DI & FE & HA & SA & SU & Total \\
\hline AN & $\mathbf{3 7}$ & 2 & 1 & 2 & 2 & 3 & 47 \\
\hline DI & 1 & $\mathbf{4 2}$ & 0 & 0 & 2 & 1 & 46 \\
\hline FE & 1 & 1 & $\mathbf{3 3}$ & 1 & 1 & 4 & 41 \\
\hline HA & 3 & 2 & 0 & $\mathbf{4 2}$ & 0 & 1 & 48 \\
\hline SA & 3 & 0 & 4 & 1 & $\mathbf{4 0}$ & 3 & 51 \\
\hline SU & 3 & 2 & 3 & 2 & 2 & $\mathbf{3 8}$ & 50 \\
\hline Total & 48 & 49 & 41 & 48 & 47 & 50 & $\mathbf{2 8 3}$ \\
\hline
\end{tabular}

Table 2: Confusion matrix for JAFFE Database

\begin{tabular}{|c|c|c|c|c|c|c|c|}
\hline $\begin{array}{c}\text { Emotions(Actual emotions row wise and } \\
\text { predicted emotions as column wise) }\end{array}$ & AN & DI & FE & HA & SA & SU & Total \\
\hline AN & $\mathbf{2 4}$ & 1 & 2 & 0 & 1 & 2 & 30 \\
\hline DI & 1 & $\mathbf{2 8}$ & 0 & 0 & 1 & 0 & 30 \\
\hline FE & 2 & 1 & $\mathbf{2 7}$ & 0 & 2 & 0 & 32 \\
\hline HA & 0 & 1 & 0 & $\mathbf{2 8}$ & 0 & 2 & 31 \\
\hline SA & 1 & 2 & 3 & 0 & $\mathbf{2 5}$ & 0 & 31 \\
\hline SU & 0 & 1 & 1 & 04 & 1 & $\mathbf{2 3}$ & 30 \\
\hline Total & 28 & 34 & 33 & 32 & 30 & 27 & $\mathbf{1 8 4}$ \\
\hline
\end{tabular}

TP- true positive, TN-true negative, FP-false positive and FN-false negative are available from confusion matrix using these values performance parameters like accuracy, FAR, FRR etc. can be calculated using following formulas.

True Positive Rate $-\mathrm{TPR}=\mathrm{TP} /(\mathrm{TP}+\mathrm{FN})$,

False Acceptance Rate $-\mathrm{FAR}=\mathrm{FPR}=\mathrm{FP} /(\mathrm{FP}+\mathrm{TN})$,

False Rejection Rate $-\mathrm{FRR}=\mathrm{FNR}=\mathrm{FN} /(\mathrm{FN}+\mathrm{TP})$,

Accuracy $=(\mathrm{TP}+\mathrm{TN}) /(\mathrm{TP}+\mathrm{TN}+\mathrm{FP}+\mathrm{FN})$

Table 3: TPR, FAR and FRR for CK+ and JAFFE Database

\begin{tabular}{|l|l|l|l|l|l|l|}
\hline \multirow{2}{*}{ Emotions } & \multicolumn{2}{|c|}{ TPR } & \multicolumn{2}{c|}{ FAR } & \multicolumn{2}{c|}{ FRR } \\
\cline { 2 - 7 } & $\begin{array}{l}\text { CK+ } \\
\text { Database }\end{array}$ & $\begin{array}{l}\text { JAFFE } \\
\text { database }\end{array}$ & $\begin{array}{l}\text { CK+ } \\
\text { Database }\end{array}$ & $\begin{array}{l}\text { JAFFE } \\
\text { database }\end{array}$ & $\begin{array}{l}\text { CK+ } \\
\text { Database }\end{array}$ & $\begin{array}{l}\text { JAFFE } \\
\text { database }\end{array}$ \\
\hline AN & 78.72 & 80.00 & 5.33 & 2.96 & 21.27 & 20.00 \\
\hline DI & 91.30 & 93.33 & 3.55 & 4.51 & 8.69 & 6.66 \\
\hline FE & 80.84 & 84.37 & 3.86 & 4.47 & 19.51 & 15.62 \\
\hline HA & 87.50 & 90.32 & 3.06 & 3.05 & 12.5 & 9.67 \\
\hline SA & 78.43 & 80.64 & 3.51 & 3.70 & 21.56 & 19.35 \\
\hline SU & 76.00 & 76.66 & 5.82 & 2.94 & 24.00 & 23.33 \\
\hline Average Value & $\mathbf{8 2 . 0 7}$ & $\mathbf{8 4 . 2 2}$ & $\mathbf{4 . 1 8}$ & $\mathbf{3 . 6 0}$ & $\mathbf{1 7 . 9 2}$ & $\mathbf{1 5 . 7 7}$ \\
& & & & & & \\
\hline
\end{tabular}

Average accuracy obtained for CK+ database is $82.07 \%$ and for JAFFE database is $84.22 \%$. 
Class wise accuracy, FAR and FRR are depicted graphically in Fig. 6, Fig. 7 and Fig. 8 respectively.

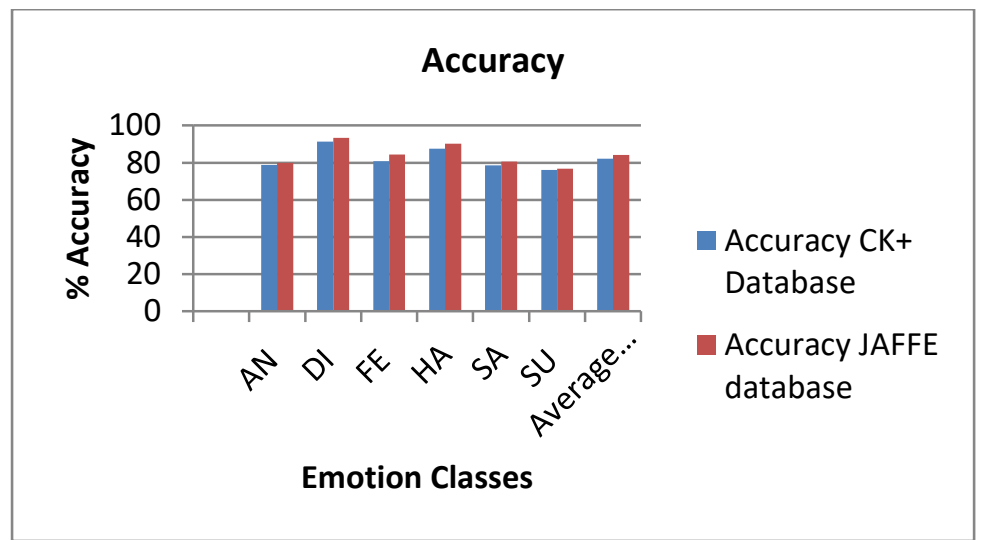

Fig. 6: Emotion Class wise performance parameter - Accuracy.
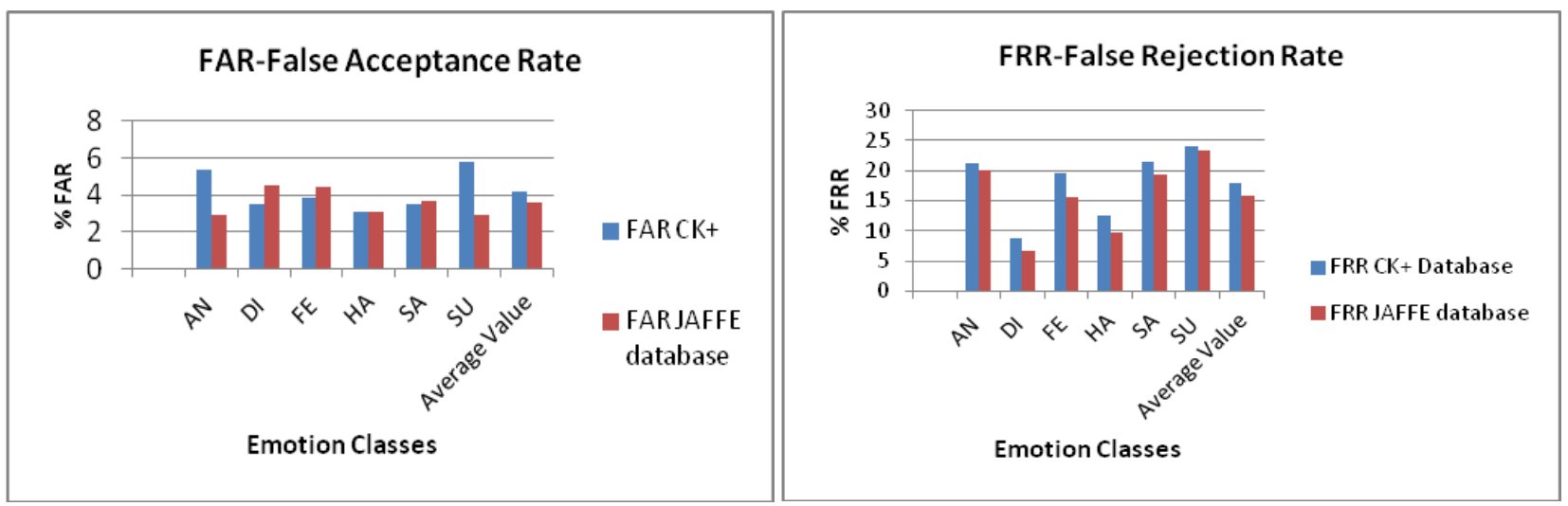

Fig.7: Emotion Class wise performance parameter - FAR Fig. 8: Emotion Class wise performance parameter - FRR.

The system can be used for any application based on emotion recognition. Emotion recognition has variety of offline and real time applications.

\section{Conclusion}

An emotion recognition system is implemented using LDN and PCA based feature extraction and FFNN-feed forward neural network as classifier. Publically available popular emotion databases are used to carry out experimentation. The emotion recognition accuracy obtained for $\mathrm{CK}+$ database is $82.07 \%$ and for JAFFE database is $84.22 \%$. FAR - false acceptance rate and FRR - false rejection rate obtained for CK+ database is $4.18 \%$ and 17.92 respectively. FAR and FRR obtained for JAFFE database is $3.60 \%$ and 15.77 respectively.

Limitation of the work carried out is, it does not address rotation and illumination variation in specific. Publically available database contains frontal face images so experimentation with pose variation is not experimented under this study however it can be experimented as future work.

As a future work accuracy can be improved and FAR and FRR can be reduced using different feature extraction methods and other than FFNN classifier. Rotation, illumination and pose variation can also be experimented.

\section{References}

[1] Adin Ramirez Rivera and Oksam Chae. Local Directional Number Pattern for Face Analysis: Face and Expression Recognition. IEEE Transactions On Image Processing. Vol. 22 , No. 5, pp. 1740-1752, May 2013

[2] Li Zhang. Intelligent facial emotion recognition using moth-firefly optimization. Elsevier Publication of International Journal of knowledge based systems. Vol 11 No. 1, pp. 248-267, Jan. 2016 
[3] Ligang Zhang. Facial Expression Recognition Using Facial Movement Features. IEEE Transactions On Affective Computing Vol 2, No. 4, pp. 219-229, April 2011

[4] Sailee R Salkar, Nikhil S Patankar, Rameshwar D Chintamani, Yogesh S Deshmukh. Illumination Invariant Face Recognition using Local Directional Number Pattern (LDN). International Journal of Engineering Development and Research. vol 5, No. 2, pp. 303-308, 2017

[5] Happy S. L. and Aurobinda Routray. Automatic facial expression recognition using features of salient facial patches. IEEE transactions on Affective Computing vol 6 No.1, 1-12, 2015.

[6] Anima Majumder. Emotion recognition from geometric facial features using self-organizing map. in special issue of Handwriting Recognition and other PR Applications. Elsevier Publications. 2013

[7] Junkai Chen, Zenghai Chen, Zheru Chi. Facial Expression Recognition in Video with Multiple Feature Fusion. IEEE Transactions on Affective Computing. PP.1-13, 2016

[8] Patrick Lucey, Jeffrey F. Cohn, Takeo Kanade, Jason Saragih, Zara Ambadar,. The Extended Cohn-Kanade Dataset $(C K+)$ : A complete dataset for action unit and emotion-specified expression. pp. 94-101, 2010.

[9] Standard Dataset-JAFFE. Available: http://www.kasrl.org/jaffe_download.html

[10] Standard Dataset - CK+. Available: http://vasc.ri.cmu.edu/idb/html/face/facial_expression/ 